\title{
Non-Hebbian Synaptic Plasticity Induced by Repetitive Postsynaptic Action Potentials
}

\author{
Hiroyuki K. Kato, ${ }^{1}$ Ayako M. Watabe, ${ }^{1,2}$ and Toshiya Manabe ${ }^{1,2}$ \\ ${ }^{1}$ Division of Neuronal Network, Department of Basic Medical Sciences, Institute of Medical Science, University of Tokyo, Tokyo 108-8639, Japan, and \\ ${ }^{2}$ Core Research for Evolutional Science and Technology, Japan Science and Technology Agency, Kawaguchi 332-0012, Japan
}

\begin{abstract}
Modern theories on memory storage have mainly focused on Hebbian long-term potentiation (LTP), which requires coincident activation of presynaptic and postsynaptic neurons for its induction. In addition to Hebbian LTP, the roles of non-Hebbian plasticity have also been predicted by some neuronal network models. However, still only a few pieces of evidence have been presented for the presence of such plasticity. In this study, we show in mouse hippocampal slices that LTP can be induced by postsynaptic repetitive depolarization alone in the absence of presynaptic inputs. The induction was dependent on voltage-dependent calcium channels instead of NMDA receptors (NMDARs), whereas the expression mechanism was shared with conventional NMDAR-dependent LTP. During the potentiation, the amplitude of spontaneous EPSCs was increased, suggesting a novel neuron-wide nature of this form of LTP. Furthermore, we also successfully induced LTP with trains of action potentials, which supported the possible existence of depolarizing pulse-induced LTP in vivo. Based on these findings, we suggest a model in which neuron-wide LTP works in concert with synapse-specific Hebbian plasticity to help information processing in memory formation.
\end{abstract}

\section{Introduction}

Since its discovery, long-term potentiation (LTP) of synaptic transmission has been regarded as the neuronal basis underlying memory formation (Bliss and Lømo, 1973; Bliss and Collingridge, 1993). In the CA1 region of the hippocampus, LTP induction has been supposed to require the coincident activation of presynaptic and postsynaptic neurons, as described in the Hebbian hypothesis (Hebb, 1949; Bliss and Collingridge, 1993). Molecular mechanisms of LTP have been studied extensively in this region, and experiments with chelators and caged compounds have directly shown $\mathrm{Ca}^{2+}$-triggered enhancement of synaptic transmission (Lynch et al., 1983; Malenka et al., 1988). Especially, the importance of $\mathrm{Ca}^{2+}$ influx through NMDA receptors (NMDARs) has been emphasized by pharmacological and genetic studies (Collingridge et al., 1983; Morris et al., 1986; Sakimura et al., 1995). NMDARs, with their characteristics as a coincident detector, are widely accepted as the molecular basis of Hebbian LTP (Dingledine et al., 1999). In contrast, the role of $\mathrm{Ca}^{2+}$ influx through voltage-dependent calcium channels (VDCCs) has not been fully addressed yet. It is well established that neuronal depolarization triggers large neuron-wide $\mathrm{Ca}^{2+}$ influx through VDCCs (Tsien et al., 1988; Jaffe et al., 1992; Nakamura et al.,

Received Dec. 11, 2008; revised July 23, 2009; accepted July 25, 2009.

This work was supported by Grants-in-Aid for Scientific Research (A.M.W. and T.M.); by the Center for Brain Medical Science, 21st Century Centers of Excellence (COE) Program (H.K.K. and T.M.); and by the Comprehensive Center of Education and Research for Chemical Biology of the Diseases, Global COE Program (T.M.) from the Ministry of Education, Science, Sports, Culture, and Technology of Japan; and by grants from The Uehara Memorial Foundation (T.M.), The Ichiro Kanehara Foundation (A.M.W.), and The Novartis Foundation (Japan) for the Promotion of Science (T.M.). We are grateful to Shizuka Kobayashi and Chieko Tazuke for helpful comments on this manuscript.

Correspondence should be addressed to Dr. Toshiya Manabe at the above address. E-mail: tmanabe-tky@ umin.ac.jp.

DOI:10.1523/JNEUROSCI.5881-08.2009

Copyright $\odot 2009$ Society for Neuroscience $\quad 0270-6474 / 09 / 2911153-08 \$ 15.00 / 0$
1999). Along with the reports on the role of VDCCs in spatial memory formation (Borroni et al., 2000; Moosmang et al., 2005), this property has led to the assumption that VDCCs can work as a trigger of non-Hebbian LTP, which induces potentiation with postsynaptic activation alone (Kullmann et al., 1992). Data supporting this idea have been presented in certain experimental conditions (Grover and Teyler, 1990; Aniksztejn and Ben-Ari, 1991; Huang and Malenka, 1993; Volgushev et al., 1994). However, many efforts to induce LTP with postsynaptic depolarization alone have not been successful in physiological conditions (Kullmann et al., 1992; Huang and Malenka, 1993; Wyllie and Nicoll, 1994; Wyllie et al., 1994; Chen et al., 1998), leading to the postulation that $\mathrm{Ca}^{2+}$ influx through VDCCs might be different in nature from that associated with the LTP induction (Guthrie et al., 1991; Müller and Connor, 1991).

In this study, using acute hippocampal slices, we found that LTP could be induced with postsynaptic depolarization alone. This potentiation was dependent on L-type VDCC (L-VDCC) activation, was occluded by NMDAR-dependent LTP, and was induced in a neuron-wide manner. Furthermore, we also successfully induced LTP with physiological activity [i.e., action potential (AP) trains]. Our results demonstrate possible involvement of VDCCs in the induction of non-Hebbian LTP in physiological conditions.

\section{Materials and Methods}

Animals. This research was approved by the Animal Care and Experimentation Committee of the University of Tokyo, and all experiments were performed according to the guidelines of the committee. C57BL/6J mice (6-10 weeks old; male) and Hartley guinea pigs ( 4 weeks old; male) were used in this study.

Solutions. The external solution contained $119 \mathrm{~mm} \mathrm{NaCl}, 2.5 \mathrm{~mm} \mathrm{KCl}$, $1.3 \mathrm{~mm} \mathrm{MgSO}_{4}, 4.0 \mathrm{~mm} \mathrm{CaCl}_{2}, 1.0 \mathrm{~mm} \mathrm{NaH}_{2} \mathrm{PO}_{4}, 26.2 \mathrm{~mm} \mathrm{NaHCO}_{3}$, $11 \mathrm{~mm}$ glucose, $100 \mu \mathrm{M}$ picrotoxin, and $50 \mu \mathrm{M} \mathrm{D}-(-)$-2-amino-5-phos- 
phonovaleric acid (D-APV), unless stated otherwise. The pairing experiments were conducted in the absence of D-APV; the miniature EPSC (mEPSC) experiments were conducted in the presence of $0.5 \mu \mathrm{m}$ tetrodotoxin (TTX), and D-APV was reduced to $25 \mu \mathrm{M}$. The internal solution contained (in $\mathrm{mm}$ ) 122.5 Csgluconate, $17.5 \mathrm{CsCl}, 8 \mathrm{NaCl}, 10 \mathrm{HEPES}, 0.2$ EGTA, $2 \mathrm{Mg}$-ATP, and $0.3 \mathrm{Na}_{3}$-GTP (pH 7.2, 290-300 mOsm). The ATP-regenerating solution contained $105 \mathrm{~mm}$ Cs-gluconate, $17.5 \mathrm{~mm}$ $\mathrm{CsCl}, 8 \mathrm{~mm} \mathrm{NaCl}, 10 \mathrm{~mm}$ HEPES, $0.2 \mathrm{~mm}$ EGTA, 2 mм Mg-ATP, 2 mм Na 2 -ATP, $0.3 \mathrm{~mm} \mathrm{Na}_{3}-$ GTP, $20 \mathrm{~mm}$ phosphocreatine, and $50 \mathrm{U} / \mathrm{ml}$ creatine phosphokinase ( $\mathrm{pH} 7.2,290-300 \mathrm{mOsm}$ ). In the experiments for AP-induced LTP, the internal solution and ATP-regenerating solution were identical to those described above, except that $\mathrm{Cs}^{+}$was substituted with $\mathrm{K}^{+}$.

Electrophysiology. Acute hippocampal slices (Bongsebandhu-phubhakdi and Manabe, 2007) were superfused continuously at a rate of 1.7$1.9 \mathrm{ml} / \mathrm{min}$ with the external solution saturated with $95 \% \mathrm{O}_{2}$ and $5 \% \mathrm{CO}_{2}$ in the submergetype recording chamber. All the experiments were performed at $25 \pm 2^{\circ} \mathrm{C}$, unless stated otherwise. To exclude epileptiform activity from the CA3 region, a cut was made between the $\mathrm{CA} 1$ and $\mathrm{CA} 3$ regions. Whole-cell patch-clamp recordings were made from CA1 pyramidal cells with the blind technique. The tips of electrodes were filled with the internal solution and backfilled with the ATP-regenerating solution. The resistance of glass electrodes was 3.5-6.5 $\mathrm{M} \Omega$. The membrane potential of the recorded cell was held at $-80 \mathrm{mV}$, unless stated otherwise. Membrane potential values were not corrected for the liquid junction potential. Recordings were made using a MultiClamp 700B amplifier (Molecular Devices). The signal was filtered at $4 \mathrm{kHz}$, digitized at $10 \mathrm{kHz}$, and stored in a personal computer equipped with Clampex 9 (Molecular Devices). For recording the baseline response, electrical stimuli were delivered at $0.1 \mathrm{~Hz}$ through a bipolar tungsten stimulating electrode placed in the stratum radiatum (see Fig. 1A). In some experiments, paired stimuli (200 ms interval) were used instead of the single stimulus. In the experiments for AP-induced LTP, the stimulating electrode was placed near the stratum pyramidale to stimulate the synapses on the proximal dendrite. The stimulus strength was adjusted to evoke EPSCs of $\sim 100$ pA. Stimulation was interrupted during the depolarizing pulses and AP trains. The series resistance and input resistance were monitored throughout the experiment by applying $5 \mathrm{mV}$ hyperpolarizing test pulses. The series resistance was typically in the range of 15-30 $\mathrm{M} \Omega$ and was not compensated to maintain the signal-to-noise ratio as high as possible. The data from the cells that deviated from any of the following criteria were discarded: (1) the resting membrane potential must be more negative than $-60 \mathrm{mV}$ at the beginning of whole-cell recordings; (2) the input resistance must be higher than $120 \mathrm{M} \Omega$ at the beginning of whole-cell recordings, except in Figure 8, in which the input resistance was typically $\sim 120$ $\mathrm{M} \Omega$; (3) the holding current must not exceed 200 pA during the baseline ${ }^{* *} p<0.005,{ }^{*} p<0.05$.
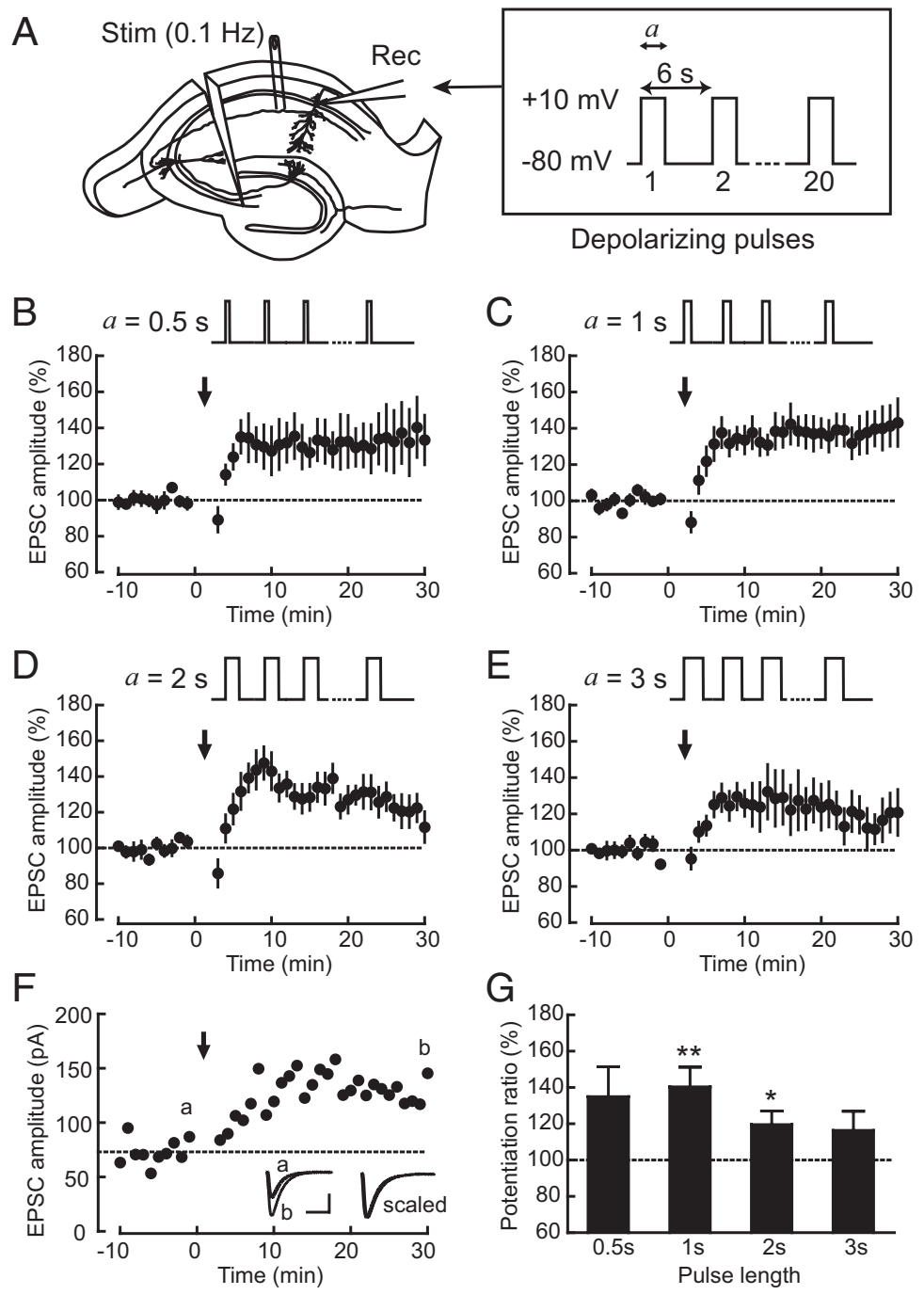

Figure 1. Repeated application of depolarizing pulses induces enduring potentiation. $A, A$ schematic diagram of experimental procedures. Rec, Recording electrode; Stim, stimulating electrode. $\boldsymbol{B}$, The averaged time course of eEPSCs in the experiments in which $0.5 \mathrm{~s}$ depolarizing pulses (arrow) were applied $(n=9)$. C, Potentiation induced by 1 s depolarizing pulses (arrow; $n=12$ ). D, Potentiation induced by 2 s depolarizing pulses (arrow; $n=9$ ). $\boldsymbol{E}$, Potentiation induced by 3 s depolarizing pulses (arrow; $n=7$ ). $\boldsymbol{F}$, A representative example of $L T P$ induced by $1 \mathrm{~s}$ depolarizing pulses (arrow). The insets show averaged EPSCs recorded at the times indicated by a and $b$ and those scaled by amplitude (scaled). Calibration: $50 \mathrm{pA}, 20 \mathrm{~ms}$. G, Summary of the potentiation by depolarizing pulses with various duration.
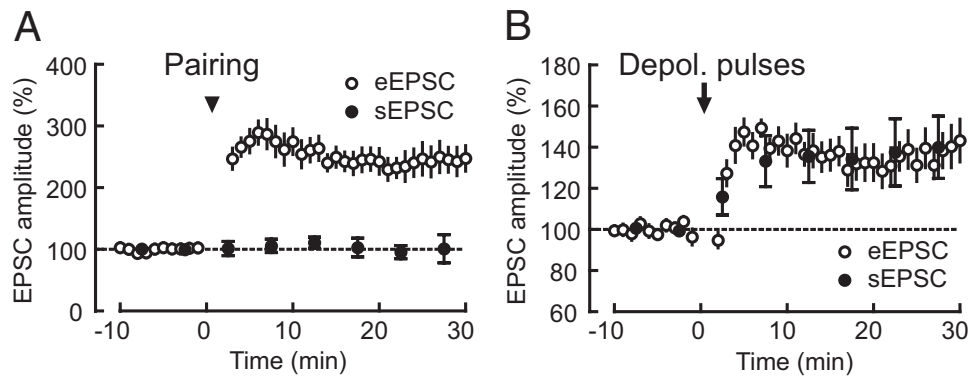

Figure 2. Non-Hebbian LTP is induced by depolarizing pulses. $\boldsymbol{A}$, The comparison of eEPSCS (open circles) and sEPSCS (filled circles) in the pairing-induced LTP experiments (Pairing, arrowhead; $n=7$ ). $\boldsymbol{B}$, The comparison of eEPSCs and sEPSCs in the experiments for depolarizing pulse-induced LTP (Depol. pulses, arrow; $n=10$ ).

period, except in Figure 7, in which it must not exceed 150 pA, and in Figure 8 , in which it must remain within $\pm 50 \mathrm{pA}$; (4) the series resistance must not change $>20 \%$ throughout the experiment; (5) conditioning must be applied within 20 min after the beginning of whole-cell record- 
A

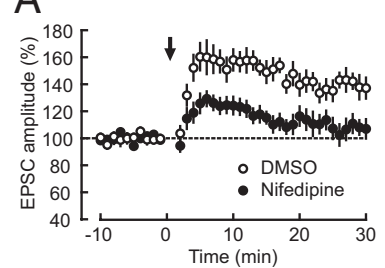

C

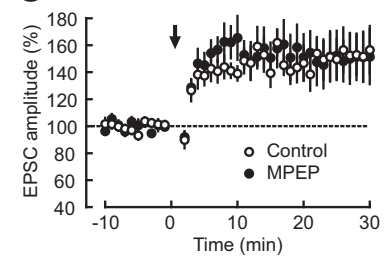

Figure 3. Pharmacological characterization of depolarizing pulse-induced LTP. $A$, Depolarizing pulse (arrow)-induced LTP in the presence of nifedipine, an L-VDCC blocker (filled circles; $n=9$ ), and DMSO alone (open circles; $n=8$ ). $\boldsymbol{B}$, Depolarizing pulse (arrow)-induced LTP in the presence of KN-93, a CaMKII inhibitor (filled circles; $n=14$ ), and KN-92, an inactive analog of KN-93 (open circles; $n=11$ ). C, Depolarizing pulse (arrow)-induced LTP in the presence (filled circles; $n=10$ ) and absence (open circles; $n=7$ ) of MPEP, an mGluR5 antagonist.
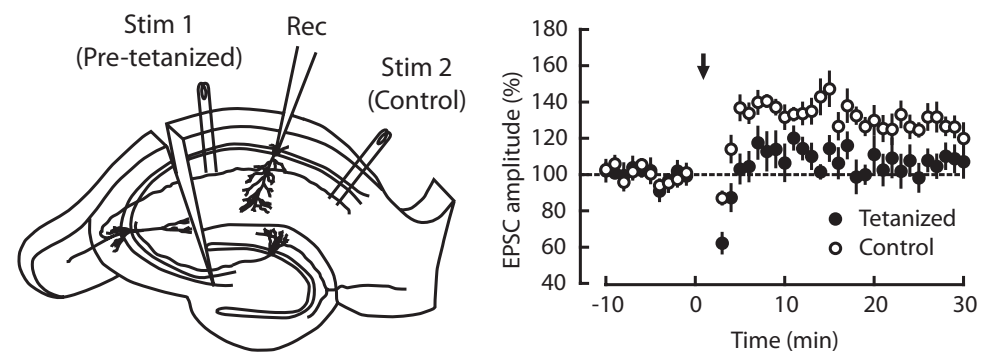

Figure 4. Depolarizing pulse-induced LTP is occluded by tetanic stimulation-induced LTP. Left, Schematic diagram of twopathway stimulation experimental procedures. A pathway for pretetanization was alternately selected from orthodromic and antidromic pathways for each experiment. Rec, Recording electrode; Stim, stimulating electrode. Right, Averaged time course of eEPSCs in the pathway tetanized beforehand (filled circles) and the control pathway (open circles) in the depolarizing pulse (arrow)-induced LTP experiments $(n=8)$.

ings; and (6) in the experiments that include spontaneous EPSC (sEPSC) analysis, both evoked EPSC (eEPSC) and sEPSC amplitudes must not grow or decline during the baseline period.

Experimental conditions for Figure 8. The neurons were held at -70 $\mathrm{mV}$. The external solution was picrotoxin free, contained $2.5 \mathrm{mM} \mathrm{CaCl}_{2}$, and was maintained at $32 \pm 2^{\circ} \mathrm{C}$. To make the reversal potential for $\mathrm{GABA}_{\mathrm{A}}$ receptors near $-70 \mathrm{mV}$, the chloride concentration of the internal solution was reduced to $16 \mathrm{~mm}$ by substituting $\mathrm{K}$-gluconate for $\mathrm{KCl}$. The distance between the stimulating and recording electrodes was kept $>500 \mu \mathrm{m}$ to reduce the contamination of $\mathrm{GABA}_{\mathrm{A}}$ synaptic currents by avoiding direct stimulation of GABAergic interneurons. At the end of each experiment, $100 \mu \mathrm{M}$ picrotoxin was applied to measure the contaminated $\mathrm{GABA}_{\mathrm{A}}$ synaptic currents, and the data were rejected if the amplitude of synaptic responses changed by $>20 \%$ of the baseline value.

LTP induction protocols. LTP was induced by four protocols: (1) for depolarizing pulse-induced LTP, the cell was depolarized repetitively to $+10 \mathrm{mV}$ for $1 \mathrm{~s} 20$ times at a 6 s interval; (2) for LTP induced by a pairing protocol, afferent fibers were stimulated 120 times at $2 \mathrm{~Hz}$ while the cell was depolarized to $0 \mathrm{mV}$; (3) in the occlusion experiment, NMDARdependent LTP was induced by five trains of tetanus $(100 \mathrm{~Hz}, 1 \mathrm{~s})$ at 3 min intervals; and (4) for AP-induced LTP, an AP train was evoked 20 times at $0.167 \mathrm{~Hz}$ in the current-clamp mode, in which each train consisted of $100 \mathrm{APs}$ at $100 \mathrm{~Hz}$ and each AP was triggered by injecting a $2 \mathrm{nA}$ current for $3 \mathrm{~ms}$. LTP was induced after obtaining $10 \mathrm{~min}$ stable baseline responses, except the experiments shown in Figures 5 and 6, in which the baseline was 5 min long. The EPSC amplitude is shown as the ratio relative to the baseline level, unless stated otherwise. The potentiation ratio was calculated as the averaged value from 26 to $30 \mathrm{~min}$ after the conditioning.

Analysis of the sEPSC and mEPSC. In sEPSC experiments, spontaneous events were detected from each trace of $150 \mathrm{~ms}$ duration that did not include eEPSCs or test pulses by using a computer program for semiautomatic detection (Mini Analysis 6.0.3; Synaptosoft) with the threshold level of $4 \mathrm{pA}$. All events larger than this level were included if they had a shape reminiscent of an EPSC and did not arise from the decaying phase of the preceding event. In mEPSC experiments, each trace was recorded every $30 \mathrm{~s}$, and events were detected from a $15 \mathrm{~s}$ window of each trace that did not include stimulus artifacts or test pulses, using the same program as the sEPSC analysis. Although the events arising from the decaying phase of the preceding events were excluded from the analysis of the MEPSC amplitude, they were included in the analysis of the mEPSC frequency.

Statistical analysis. All values are expressed as the mean \pm SEM. Statistical analysis was performed using unpaired Student's $t$ test, except the comparison between eEPSCs and sEPSCs and the comparison between rise times, in which the paired $t$ test was applied.

Drugs. Drugs used in this study were D-APV, 2-methyl-6-(phenylethynyl)pyridine (MPEP), nifedipine (Tocris Bioscience), KN-92, KN-93 (Calbiochem), picrotoxin (Sigma-Aldrich), and TTX (SANKYO).

\section{Results}

Neuron-wide LTP induced by repeated postsynaptic depolarization

We tested the effect of VDCC activation on EPSCs by applying depolarizing pulses to the voltage-clamped pyramidal cells in the presence of the NMDAR blocker D-APV (25-50 $\mu \mathrm{M})$. Previously, we have shown in the young guinea pig that repeated application of depolarizing pulses leads to transient potentiation of EPSCs (Kullmann et al., 1992). After confirming the result (Fig. S1, available at www.jneurosci.org as supplemental material), we modified the protocols to improve the efficiency of VDCC activation (Fig. 1), using acute hippocampal slices from adult mice. We systematically changed the duration of the depolarizing pulses to $0.5,1,2$, and $3 \mathrm{~s}$ (Fig. 1A-E). As summarized in Figure $1 G$, significant potentiation of eEPSC was induced when the duration was 1 and $2 \mathrm{~s}(0.5 \mathrm{~s}: 135.0 \pm 16.4 \%, n=9, p=0.0657 ; 1 \mathrm{~s}$ : $140.3 \pm 11.0 \%, n=12, p=0.00363 ; 2 \mathrm{~s}: 119.6 \pm 7.5 \%, n=9, p=$ $0.0311 ; 3 \mathrm{~s}: 116.3 \pm 10.6, n=7, p=0.174)$. Since this form of LTP was induced with postsynaptic repetitive depolarization alone, this result suggested the possibility of nonspecific potentiation in all synapses over the depolarized neuron, regardless of the presynaptic activity at these synapses. This is in contrast to Hebbian LTP, which is induced almost exclusively in synapses that receive inputs during LTP induction (Nicoll et al., 1988).

To examine the nature of this neuron-wide LTP in detail, we next analyzed sEPSCs, using the pulse duration of $1 \mathrm{~s}$. Because sEPSCs occur randomly throughout the whole dendrite, its average amplitude would be increased only if LTP is induced in the majority of synapses, and thus the sEPSC amplitude could be a good measure of neuron-wide changes in the synaptic efficacy (Wyllie et al., 1994). Based on this property of sEPSCs, we compared their behavior between NMDAR-dependent Hebbian LTP and depolarizing pulse-induced LTP (Fig. 2). To induce NMDAR-dependent LTP, depolarization of the recorded cell was paired with afferent fiber stimulation in the absence of D-APV. Although robust LTP of eEPSCs was induced (245.5 \pm $22.2 \% ; n=7 ; p=0.000611)$, the amplitude of sEPSCs $(100.9 \pm$ $22.9 \% ; p=0.969$ ), which were simultaneously recorded from the same cell, failed to show any significant change (Fig. 2A). The 
A

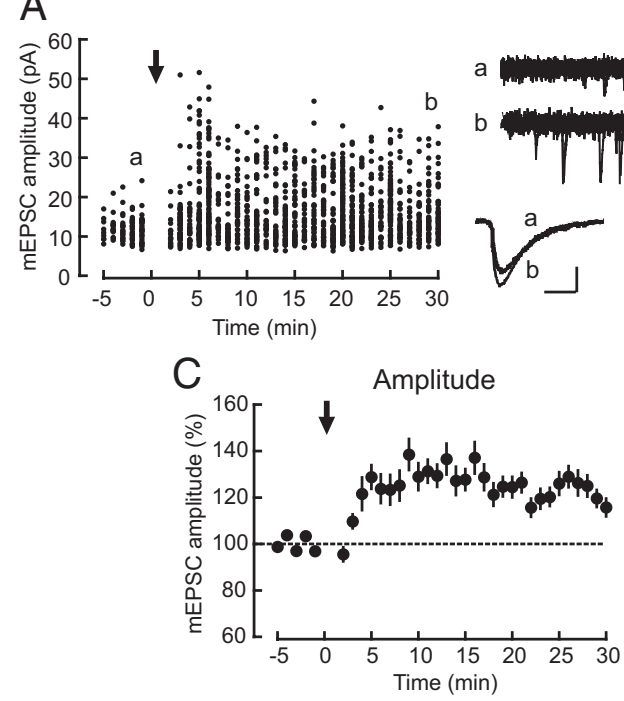

$\mathrm{B}$
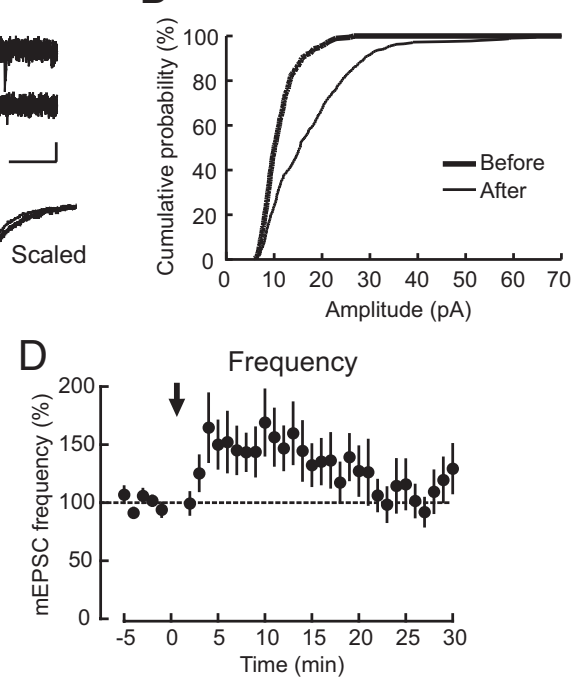

Figure 5. Potentiation of mEPSCs in depolarizing pulse-induced LTP. A, Left, Representative plot of mEPSC LTP by depolarizing pulses (arrow). Right, Top, Ten consecutive sweeps before (a) and after (b) depolarizing pulses are superimposed. Calibration: 10 pA, $200 \mathrm{~ms}$. Right, Bottom, Averaged mEPSCs recorded at the times indicated by a and b and those scaled by amplitude (Scaled). Calibration: 5 pA, $10 \mathrm{~ms}$. B, A representative cumulative plot of mEPSC amplitudes before (thick line) and after (thin line) depolarizing pulses. $\boldsymbol{C}$, The averaged time course of mEPSC amplitudes in the experiments for mEPSC LTP $(n=17)$. D, The averaged time course of mEPSC frequency in the experiments for mEPSC LTP.

potentiation ratio was significantly different between eEPSCs and sEPSCs $(p=0.000331)$. This result is in accord with the notion that sEPSCs are not sensitive enough to detect the change in a small subset of synapses if no attempt was made to identify sEPSCs from those synapses (Manabe et al., 1992). In contrast, depolarizing pulse-induced LTP of eEPSCs $(138.5 \pm 8.5 \% ; n=$ $10 ; p=0.00142$ ) (Fig. $2 B$ ) was accompanied by the equivalent $(p=0.944)$ increase in the amplitude of sEPSCs (140.0 $\pm 15.1 \%$; $p=0.0297)$ recorded from the same neuron, which strongly suggested a neuron-wide change of the EPSC amplitude. These results support the non-Hebbian characteristics of depolarizing pulse-induced LTP.

\section{Characterization of depolarizing pulse-induced LTP}

To elucidate the molecular mechanism, we examined pharmacological properties of depolarizing pulse-induced LTP. As shown in Figure $3 A$, the L-VDCC blocker nifedipine $(20 \mu \mathrm{M})$ significantly ( $p=0.00257$ ) inhibited the potentiation [dimethyl sulfoxide (DMSO) control: $140.8 \pm 6.6 \%, n=8, p=0.000460$; nifedipine, $107.0 \pm 6.6 \%, n=9, p=0.316]$, indicating the contribution of L-VDCCs to depolarizing pulse-induced LTP. We next tested the effect of the calcium/calmodulindependent protein kinase II (CaMKII) inhibitor KN-93, since CaMKII has been regarded as an essential enzyme for NMDAR-dependent LTP (Lisman, 1989; Malenka et al., 1989; Malinow et al., 1989; Silva et al., 1992). As shown in Figure 3B, KN-93 (5 $\mu \mathrm{M})$ significantly $(p=0.0309)$ reduced the potentiation ratio $(122.9 \pm 7.3 \% ; n=14 ; p=0.00809)$ compared with its inactive analog KN-92 (151.2 $\pm 9.8 \% ; n=11 ; p=0.000380)$, suggesting that depolarizing pulse-induced LTP was at least partially dependent on CaMKII and shared the induction and/or expression mechanism with NMDAR-dependent LTP. The potentiation remaining under KN-93 is likely to be attributable to incomplete blockade of CaMKII, but additional contribution of other $\mathrm{Ca}^{2+}$-activated enzymes cannot be denied. We also examined possible contribution of the metabotropic glutamate receptor (mGluR) to this potentiation. We have previously shown cooperative induction of LTP by VDCC activation and synaptic inputs in the presence of an NMDAR antagonist (Kullmann et al. 1992), which suggests possible involvement of mGluR activation by afferent stimulation in this form of LTP. Although synaptic stimulation was dispensable for LTP induction in the present study, there remains the possibility that stimulus-independent ambient glutamate could have activated mGluRs. As mGluR5 is the major, if not only, subtype reported to be expressed in pyramidal cells (Shigemoto et al., 1993), we used the mGluR5-selective antagonist MPEP. As shown in Figure 3C, the potentiation ratio was indistinguishable $(p=0.885)$ between MPEP $(4 \mu \mathrm{M})$-treated $(150.4 \pm 17.1 \% ; n=10 ; p=0.0259)$ and control $(154.0 \pm 7.0 \% ; n=$ $7 ; p=0.0100)$ slices. Together, $\mathrm{Ca}^{2+}$ influx triggered by depolarizing pulses is sufficient for LTP induction, and neither synaptic stimulation nor tonic mGluR activation is needed for depolarizing pulseinduced LTP.

After the LTP induction, we observed a small but significant reduction in the paired-pulse ratio (PPR) $(94.1 \pm 1.9 \% ; n=25$; $p=0.00542$ ) (Fig. S2, available at www.jneurosci.org as supplemental material), which may reflect some change in the presynaptic terminals. However, the reduction was rather small, and the most part of the reduction could be explained by the nonlinearity of the recording; thus it is unlikely that presynaptic mechanisms alone can fully account for the observed potentiation. The little change in the PPR and the requirements for CaMKII lead to the possibility that this type of LTP shares mechanisms with NMDAR-dependent LTP. We therefore determined whether depolarizing pulse-induced LTP was reduced by prior induction of NMDAR-dependent LTP. Two pathways were stimulated in this experiment (Fig. 4), and LTP was induced only in one pathway with five trains of tetanus $(100 \mathrm{~Hz}, 1 \mathrm{~s})$ in the absence of D-APV, whereas the other pathway served as the control. After the application of the tetani, whole-cell recordings were performed, in which both pathways were monitored throughout the following experiment for depolarizing pulse-induced LTP in the same slice. Although LTP was consistently induced in the control pathway (127.2 $\pm 5.4 \% ; n=8 ; p=0.00143)$, the tetanized pathway failed to show potentiation $(107.7 \pm 5.6 \%$; $p=0.209)$. The potentiation ratio was significantly different between the pathways ( $p=$ 
A

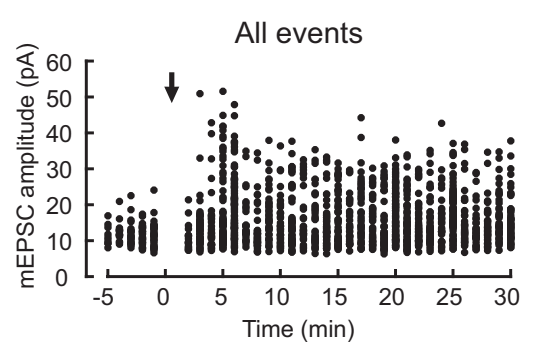

$\mathrm{B}$

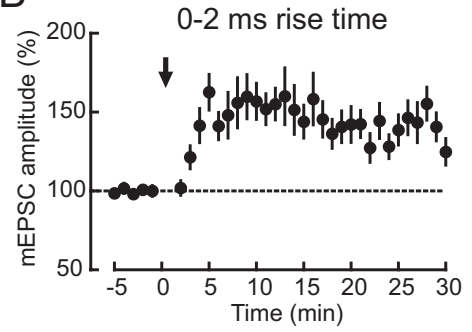

$\mathrm{D}$

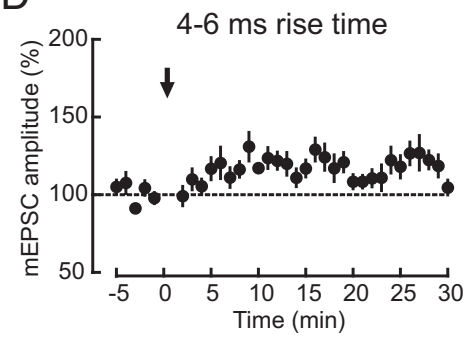

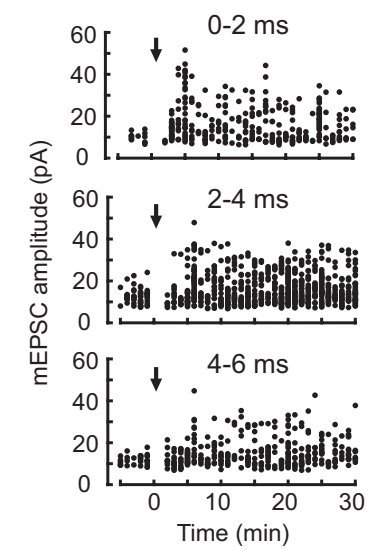

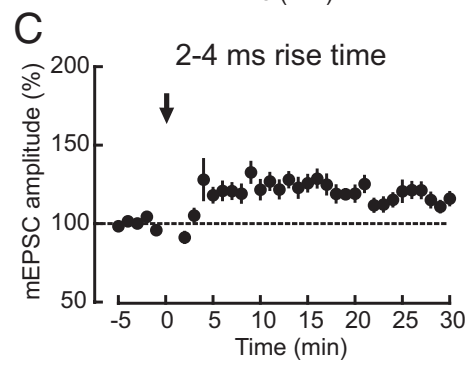

E

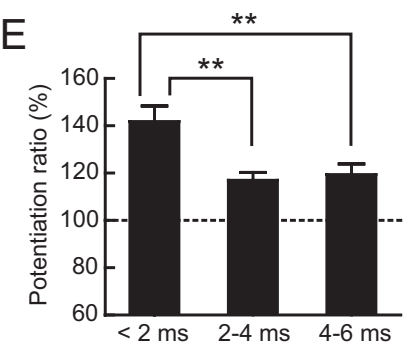

Figure 6. The mEPSC with a fastertime course is potentiated more during depolarizing pulse-induced LTP.A, An example of the sorting of $\mathrm{mEPSC}$ by their rise times. Left, A plot of the amplitude of all miniature events in the experiments for depolarizing pulse (arrow)-induced LTP. Right, Plots of the amplitude of miniature events with the 10-90\% rise time of $0-2 \mathrm{~ms}$ (top), 2-4 ms (middle), and 4-6 ms (bottom). $\boldsymbol{B}-\boldsymbol{D}$, The averaged time course of the amplitude of $\mathrm{mEPSC}$, whose rise time is in the range of $0-2 \mathrm{~ms}(\boldsymbol{B}), 2-4 \mathrm{~ms}(\boldsymbol{C})$, or $4-6$ $\mathrm{ms}(\boldsymbol{D})$. $\boldsymbol{E}$, Summary of the potentiation ratio in the three groups shown in $\boldsymbol{B}-\boldsymbol{D} .{ }^{* *} p<0.005$ (paired $t$ test).

0.00725 ), indicating the occlusion of potentiation by the prior induction of NMDAR-dependent LTP. This result clearly shows the sharing of mechanisms between depolarizing pulse-induced and NMDAR-dependent LTP.

\section{mEPSC potentiation by depolarizing pulses}

Although sEPSCs may consist mainly of mEPSCs that represent quantal minimal responses at the synapses, they may contain EPSCs evoked by spontaneous activities of afferent fibers. To confirm the increase in quantal size in depolarizing pulseinduced LTP, we next applied TTX, a sodium channel blocker, to block APs and examined the behavior of mEPSCs during the potentiation (Manabe et al., 1992). This analysis also enabled us to characterize the potentiation in more detail because of the much larger number of events detected in mEPSC experiments than in sEPSC experiments. Figure $5 A$ shows a representative example, in which the amplitude of mEPSCs increased, following depolarizing pulses. Potentiation of the mEPSCs can be seen more clearly in a cumulative plot (Fig. $5 B$ ). The time course of the change in mEPSC amplitudes (Fig. 5C) indicated that the potentiation of mEPSCs $(123.1 \pm 3.4 \% ; n=17 ; p<0.00001)$ was long lasting as that of eEPSCs, which was also accompanied by a ten- dency of the increase in frequency (Fig. $5 D)$ although it is not statistically significant $(110.4 \pm 15.3 \%$; $p=0.507)$. The potentiation of mEPSC amplitudes indicates an increase in quantal size, supporting the postsynaptic expression of depolarizing pulse-induced LTP. The tendency of an increase in mEPSC frequency might indicate some contribution of presynaptic change, but it can also be explained solely by postsynaptic mechanisms (Manabe et al., 1992).

Because it has been reported that the distribution of L-VDCCs is biased toward the soma over the dendrite in pyramidal cells (Westenbroek et al., 1990), we next examined the correlation between the potentiation and the distance from the soma. We used the 10-90\% rise time of the initial phase of mEPSCs as a measure of the distance (Magee and Cook, 2000) and classified mEPSCs recorded in all experiments into three groups by the rise time of $0-2,2-4$, and 4-6 ms (Fig. 6A). As shown in Figure $6 B-D$, the potentiation was largest in the $0-2$ ms group. The average potentiation ratio was $141.8 \pm 6.6$, $116.9 \pm 3.4$, and $119.4 \pm 4.5 \%$ for the $0-2,2-4$, and 4-6 ms groups, respectively (Fig. 6E). There were significant differences between the 0-2 ms group and the other two groups ( $0-2$ vs $2-4 \mathrm{~ms}, p=$ $0.00194 ; 0-2$ vs $4-6 \mathrm{~ms}, p=0.00366)$. These results suggest that the potentiation induced by depolarizing pulses is larger, on average, at the synapse located close to the soma than at the synapse located far from the soma. This conclusion is in parallel with the biased distribution of L-VDCCs in favor of the soma (Westenbroek et al., 1990), although the possibility of poor space-clamping at the synapse located far from the soma in whole-cell recordings cannot be excluded (Williams and Mitchell, 2008).

\section{Neuron-wide LTP induced by AP trains}

Then, does depolarizing pulse-induced LTP take place in more physiological conditions? In living animals, oscillatory electrical activities at up to $200 \mathrm{~Hz}$ have been reported in the CA1 region during exploration behavior (Ranck, 1973; O'Keefe, 1976), which may include trains of APs in the pyramidal cells (Ranck, 1973; O'Keefe, 1976; Huxter et al., 2003). Because APs have been shown to back-propagate into the dendrites (Stuart and Sakmann, 1994) and activate VDCCs (Jaffe et al., 1992; Nakamura et al., 1999) and CaMKII (Yasuda et al., 2003), it is possible that this type of LTP could also be induced by repetitive APs in hippocampal slices. To test this hypothesis, we examined a possible impact of APs triggered in the current-clamp mode on synaptic responses in the same cell. Figure $7 A$ illustrates the protocol for the induction of LTP by trains of APs evoked in the postsynaptic cell, which imitates the depolarizing pulses used in the present study (Fig. 1A): an AP train that consisted of $100 \mathrm{APs}$ at $100 \mathrm{~Hz}$ was repeatedly applied 20 times at a $6 \mathrm{~s}$ interval. The AP trains induced enduring 

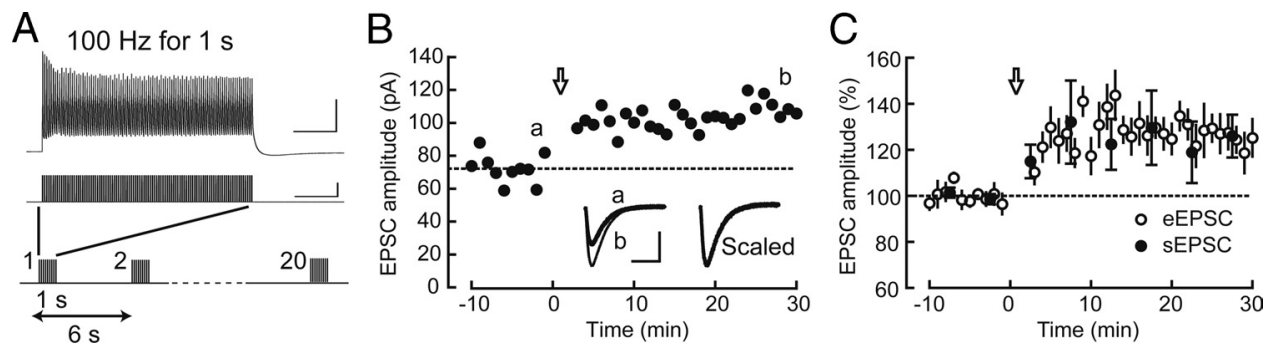

Figure 7. Repeated trains of APs induce LTP. A, The top trace shows the membrane potential during an AP train. Calibration: $50 \mathrm{mV}, 200 \mathrm{~ms}$. The middle and bottom traces indicate the injected current. Calibration: $1 \mathrm{nA}, 200 \mathrm{~ms}$. B, A representative time course of LTP induced by AP trains (open arrow). The insets show averaged EPSCs recorded at the times indicated by a and $b$ and those scaled by amplitude (Scaled). Calibration: 50 pA, 20 ms. C, The comparison of eEPSCs (open circles) and sEPSCs (filled circles) in the experiments for AP train (open arrow)-induced LTP ( $n=7$ ).

potentiation of eEPSCs that lasted at least for $30 \mathrm{~min}$ (Fig. $7 \mathrm{~B}, C)$. This LTP (124.5 \pm $6.6 \% ; n=7 ; p=0.00964)$ was accompanied by the equivalent $(p=0.851)$ increase in amplitude of sEPSCs $(126.0 \pm$ 9.3\%; $p=0.0309$ ) recorded from the same neuron, indicating that neuronwide LTP can be induced by APs as well. Finally, we confirmed that AP traininduced LTP was reproducible also in more physiological conditions (i.e., 2.5 $\mathrm{mM} \mathrm{Ca}^{2+}, 32 \pm 2^{\circ} \mathrm{C}, V_{\text {hold }}=-70 \mathrm{mV}$ and picrotoxin free) $(137.9 \pm 8.6 \% ; n=8 ; p=$ 0.00312) (Fig. 8).

\section{Discussion}

Induction of non-Hebbian LTP by

postsynaptic depolarization

Compared with the well known role of NMDAR-dependent $\mathrm{Ca}^{2+}$ influx in the induction of LTP (Collingridge et al., 1983; Morris et al., 1986; Sakimura et al., 1995), the contribution of $\mathrm{Ca}^{2+}$ influx through VDCCs has been controversial. It has been reported that tetanization with a very high frequency $(200 \mathrm{~Hz})$ or extracellular application of tetraethylammonium, a potassium channel blocker, can cause synaptic potentiation in a VDCCdependent manner (Grover and Teyler, 1990; Aniksztejn and Ben-Ari, 1991; Huang and Malenka, 1993); however, since these induction procedures were accompanied by synaptic stimulation, factors other than $\mathrm{Ca}^{2+}$ might have contributed to these forms of LTP. Efforts to induce LTP with postsynaptic depolarization alone have not been so successful: in the visual cortex, application of intracellular tetanization was reported to induce either LTP or LTD, although it is unknown how these two forms of plasticity occur in the same condition (Volgushev et al., 1994), and in the hippocampus, there has been one report of mEPSC LTP in the culture system (Baxter and Wyllie, 2006); however, more physiological experiments with acute hippocampal slices have reported only short-term potentiation so far (Kullmann et al., 1992; Huang and Malenka, 1993; Wyllie et al., 1994; Wyllie and Nicoll, 1994; Chen et al., 1998). In this study, we found a condition in which LTP is induced by postsynaptic depolarization alone, using acute hippocampal slices from adult mice. Furthermore, we successfully induced potentiation by evoking AP trains in the postsynaptic neuron, supporting the physiological relevance of this form of LTP. These results show the capability of VDCC-derived $\mathrm{Ca}^{2+}$ influx to induce LTP without NMDAR activation and suggest the possible role of VDCCs in memory formation. This view is in accord with the studies on L-VDCC subunit knock-out mice, which showed deficiency in spatial learning despite the normal induction of NMDAR-dependent LTP (Moosmang et al., 2005).

Our results have given several implications for the molecular mechanisms of depolarizing pulse-induced LTP. First, the pharmacological experiments indicate the involvement of L-VDCCs and CaMKII. Second, the potentiation in the mEPSC amplitudes suggests the postsynaptic expression. Third, depolarizing pulseinduced LTP was occluded by NMDAR-dependent LTP. Together, the most straightforward explanation is that $\mathrm{Ca}^{2+}$ influx through VDCCs can induce LTP in the same manner as $\mathrm{Ca}^{2+}$ influx through NMDARs (Lisman, 1989; Malenka et al., 1989; Malinow et al., 1989; Manabe et al., 1992; Silva et al., 1992). A contradictory report has been given by Chen et al. (1998), which suggests a novel mechanism for depolarizing pulse-induced transient potentiation, based on the decrease in the PPR and slowing of the eEPSC kinetics. However, we observed only a minor change in the PPR and also did not find any change in the kinetics of eEPSCs in our present study (Fig. S3, available at www.jneurosci.org as supplemental material). Although the mEPSC kinetics became faster after conditioning, this can be explained by the preferential potentiation of mEPSCs with a faster time course (Fig. 6). These results, along with the occlusion experiment (Fig. 4), suggest different mechanisms from those described by Chen et al. (1998) and are consistent with the notion that this type of LTP shares the expression mechanism with NMDAR-dependent LTP.

In the previous report, we have observed only transient potentiation by depolarizing pulses, which was converted into sustained potentiation by concomitant synaptic stimulation (Kullmann et al., 1992). This is in contrast with our present study, in which we could induce stable LTP by postsynaptic depolarization alone in the absence of synaptic inputs. The possible reasons for this discrepancy are as follows. First, potentiation in this study may also be a transient one, decaying in a time course longer than our observation period. However, this is quite unlikely, because we often recorded as long as nearly $1 \mathrm{~h}$ after conditioning and 
observed stable potentiation without a tendency for decay. Second, the species and age of experimental animals might account for the difference. In the present study, adult mice were used instead of 3- to 4 -week-old guinea pigs in the previous one. It is possible that some intracellular signaling cascade is constitutively active in our present preparation, thus skipping the need for synaptic stimulation. Finally, there may be a threshold in the amount of $\mathrm{Ca}^{2+}$ influx for stable potentiation. In the previous study, additional $\mathrm{Ca}^{2+}$ might have been provided in the postsynaptic cell by synaptic stimulation, possibly through mGluR activation (Finch and Augustine, 1998; Takechi et al., 1998), which may be required for stable potentiation. The modified depolarization protocol in the present study might have enabled $\mathrm{Ca}^{2+}$ influx that is sufficient to exceed the threshold without the help of synaptic inputs.

\section{Physiological relevance of non-Hebbian LTP}

The most striking characteristics that distinguish depolarizing pulse-induced LTP from NMDAR-dependent LTP are its nonHebbian nature. Neuronal network models have suggested the roles of non-Hebbian plasticity in memory formation (Poirazi and Mel, 2001; Govindarajan et al., 2006), but still only a handful of evidence has been presented for such plasticity (Alonso et al., 1990; Engert and Bonhoeffer, 1997). Previous studies have reported neuron-wide LTP induced by applying drugs such as forskolin (Otmakhov et al., 2004), but this is the first report on the neuron-wide LTP induced by physiological neuronal activity (i.e., AP trains). Although we also tried to induce LTP with APs triggered by synaptic stimulation, this attempt failed (data not shown), presumably because neurotransmitter release was rapidly attenuated during repeated activation of the same afferent fibers, which constituted a small portion of the inputs to the recorded area. Of course, this result does not exclude the possibility of AP-dependent LTP induction in vivo, in which many more synapses could be activated, compared with electrical stimulation. An important problem is that, if high-frequency firing alone is enough to induce neuron-wide strengthening of synapses, it would lead to uncontrolled growing of activity in a positive-feedback manner and eventually destabilize the neuronal network (Turrigiano and Nelson, 2000). A plausible candidate for managing this possible problem is homeostatic plasticity, a regulatory mechanism that globally adjusts synaptic strengths of all the synapses on the postsynaptic neuron to keep the overall activity in the normal range (O'Brien et al., 1998; Turrigiano et al., 1998; Turrigiano and Nelson, 2000). We propose that APinduced neuron-wide LTP might work as a substrate for memory over hours to days, which is kept under control with the help of homeostatic plasticity working in the longer time course.

The most challenging question is how this plasticity contributes to memory formation. Modern theories of memory mechanisms have almost exclusively focused on the Hebbian rule: this rule is considered advantageous in that it enlarges capacity for memory through the selective regulation of each synapse as an independent unit for computation of the information (Yuste and Denk, 1995). In contrast, our findings suggest the possible existence of neuron-wide synaptic plasticity that goes against the Hebbian rule: LTP is induced at synapses that do not receive afferent inputs, and the only prerequisite for the potentiation is that the neuron fires frequently enough. Although neuron-wide LTP would not improve information processing within a neuron per se, it could influence the output from the neuronal network as a whole. For example, it might scale up the contribution of intensely activated neurons in the network (Zhang and Linden,
2003). One intriguing hypothesis is that memory may not be stored solely by Hebbian plasticity, but that plasticity at the following two levels might contribute to memory formation in concert: the synaptic level (Hebbian plasticity) and the neuron-wide level (non-Hebbian plasticity). The orchestrated operation of these two mechanisms for plasticity might make efficient computation possible and help accomplish the wide variety of learning and memory tasks that the neuronal network performs. A detailed modeling study in future will elucidate the exact role and significance of this novel plasticity in memory formation.

\section{References}

Alonso A, De Curtis M, Llinás R (1990) Postsynaptic Hebbian and nonHebbian long-term potentiation of synaptic efficacy in the entorhinal cortex in slices and in the isolated adult guinea pig brain. Proc Natl Acad Sci U S A 87:9280-9284.

Aniksztejn L, Ben-Ari Y (1991) Novel form of long-term potentiation produced by a $\mathrm{K}^{+}$channel blocker in the hippocampus. Nature 349:67-69.

Baxter AW, Wyllie DJA (2006) Phosphatidylinositol 3 kinase activation and AMPA receptor subunit trafficking underlie the potentiation of miniature EPSC amplitudes triggered by the activation of L-type calcium channels. J Neurosci 26:5456-5469.

Bliss TV, Lømo T (1973) Long-lasting potentiation of synaptic transmission in the dentate area of the anaesthetized rabbit following stimulation of the perforant path. J Physiol 232:331-356.

Bliss TVP, Collingridge GL (1993) A synaptic model of memory: long-term potentiation in the hippocampus. Nature 361:31-39.

Bongsebandhu-phubhakdi S, Manabe T (2007) The neuropeptide nociceptin is a synaptically released endogenous inhibitor of hippocampal longterm potentiation. J Neurosci 27:4850-4858.

Borroni AM, Fichtenholtz H, Woodside BL, Teyler TJ (2000) Role of voltage-dependent calcium channel long-term potentiation (LTP) and NMDA LTP in spatial memory. J Neurosci 20:9272-9276.

Chen HX, Hanse E, Pananceau M, Gustafsson B (1998) Distinct expressions for synaptic potentiation induced by calcium through voltage-gated calcium and N-methyl-D-aspartate receptor channels in the hippocampal CA1 region. Neuroscience 86:415-422.

Collingridge GL, Kehl SJ, McLennan H (1983) Excitatory amino acids in synaptic transmission in the Schaffer collateral-commissural pathway of the rat hippocampus. J Physiol 334:33-46.

Dingledine R, Borges K, Bowie D, Traynelis SF (1999) The glutamate receptor ion channels. Pharmacol Rev 51:7-61.

Engert F, Bonhoeffer T (1997) Synapse specificity of long-term potentiation breaks down at short distances. Nature 388:279-284.

Finch EA, Augustine GJ (1998) Local calcium signalling by inositol-1,4,5trisphosphate in Purkinje cell dendrites. Nature 396:753-756.

Govindarajan A, Kelleher RJ, Tonegawa S (2006) A clustered plasticity model of long-term memory engrams. Nat Rev Neurosci 7:575-583.

Grover LM, Teyler TJ (1990) Two components of long-term potentiation induced by different patterns of afferent activation. Nature 347:477-479.

Guthrie PB, Segal M, Kater SB (1991) Independent regulation of calcium revealed by imaging dendritic spines. Nature 354:76-80.

Hebb DO (1949) The organization of behavior: a neuropsychological theory. New York: Wiley Interscience.

Huang YY, Malenka RC (1993) Examination of TEA-induced synaptic enhancement in area CA1 of the hippocampus: the role of voltage-dependent $\mathrm{Ca}^{2+}$ channels in the induction of LTP. J Neurosci 13:568-576.

Huxter J, Burgess N, O’Keefe J (2003) Independent rate and temporal coding in hippocampal pyramidal cells. Nature 425:828-832.

Jaffe DB, Johnston D, Lasser-Ross N, Lisman JE, Miyakawa H, Ross WN (1992) The spread of $\mathrm{Na}^{+}$spikes determines the pattern of dendritic $\mathrm{Ca}^{2+}$ entry into hippocampal neurons. Nature 357:244-246.

Kullmann DM, Perkel DJ, Manabe T, Nicoll RA (1992) $\mathrm{Ca}^{2+}$ entry via postsynaptic voltage-sensitive $\mathrm{Ca}^{2+}$ channels can transiently potentiate excitatory synaptic transmission in the hippocampus. Neuron 9:1175-1183.

Lisman J (1989) A mechanism for the Hebb and the anti-Hebb process underlying learning and memory. Proc Natl Acad Sci U S A 86:9574-9578.

Lynch G, Larson J, Kelso S, Barrionuevo G, Schottler F (1983) Intracellular injections of EGTA block induction of hippocampal long-term potentiation. Nature 305:719-721.

Magee JC, Cook EP (2000) Somatic EPSP amplitude is independent of syn- 
apse location in hippocampal pyramidal neurons. Nat Neurosci 3:895-903.

Malenka RC, Kauer JA, Zucker RS, Nicoll RA (1988) Postsynaptic calcium is sufficient for potentiation of hippocampal synaptic transmission. Science 242:81-84.

Malenka RC, Kauer JA, Perkel DJ, Mauk MD, Kelly PT, Nicoll RA, Waxham MN (1989) An essential role for postsynaptic calmodulin and protein kinase activity in long-term potentiation. Nature 340:554-557.

Malinow R, Schulman H, Tsien RW (1989) Inhibition of postsynaptic PKC or CaMKII blocks induction but not expression of LTP. Science 245:862-866.

Manabe T, Renner P, Nicoll RA (1992) Postsynaptic contribution to longterm potentiation revealed by the analysis of miniature synaptic currents. Nature 355:50-55.

Moosmang S, Haider N, Klugbauer N, Adelsberger H, Langwieser N, Müller J, Stiess M, Marais E, Schulla V, Lacinova L, Goebbels S, Nave KA, Storm DR, Hofmann F, Kleppisch T (2005) Role of hippocampal Cav1.2 $\mathrm{Ca}^{2+}$ channels in NMDA receptor-independent synaptic plasticity and spatial memory. J Neurosci 25:9883-9892.

Morris RGM, Anderson E, Lynch GS, Baudry M (1986) Selective impairment of learning and blockade of long-term potentiation by an N-methylD-aspartate receptor antagonist, AP5. Nature 319:774-776.

Müller W, Connor JA (1991) Dendritic spines as individual neuronal compartments for synaptic $\mathrm{Ca}^{2+}$ responses. Nature 354:73-76.

Nakamura T, Barbara JG, Nakamura K, Ross WN (1999) Synergistic release of $\mathrm{Ca}^{2+}$ from $\mathrm{IP}_{3}$-sensitive stores evoked by synaptic activation of mGluRs paired with backpropagating action potentials. Neuron 24:727-737.

Nicoll RA, Kauer JA, Malenka RC (1988) The current excitement in longterm potentiation. Neuron 1:97-103.

O’Brien RJ, Kamboj S, Ehlers MD, Rosen KR, Fischbach GD, Huganir RL (1998) Activity-dependent modulation of synaptic AMPA receptor accumulation. Neuron 21:1067-1078.

O'Keefe J (1976) Place units in the hippocampus of the freely moving rat. Exp Neurol 51:78-109.

Otmakhov N, Khibnik L, Otmakhova N, Carpenter S, Riahi S, Asrican B, Lisman J (2004) Forskolin-induced LTP in the CA1 hippocampal region is NMDA receptor dependent. J Neurophysiol 91:1955-1962.

Poirazi P, Mel BW (2001) Impact of active dendrites and structural plasticity on the memory capacity of neural tissue. Neuron 29:779-796.

Ranck JB Jr (1973) Studies on single neurons in dorsal hippocampal formation and septum in unrestrained rats. I. Behavioral correlates and firing repertoires. Exp Neurol 41:461-531.

Sakimura K, Kutsuwada T, Ito I, Manabe T, Takayama C, Kushiya E, Yagi T,
Aizawa S, Inoue Y, Sugiyama H, Mishina M (1995) Reduced hippocampal LTP and spatial learning in mice lacking NMDA receptor $\varepsilon 1$ subunit. Nature 373:151-155.

Shigemoto R, Nomura S, Ohishi H, Sugihara H, Nakanishi S, Mizuno N (1993) Immunohistochemical localization of a metabotropic glutamate receptor, mGluR5, in the rat brain. Neurosci Lett 163:53-57.

Silva AJ, Stevens CF, Tonegawa S, Wang Y (1992) Deficient hippocampal long-term potentiation in $\alpha$-calcium-calmodulin kinase II mutant mice. Science 257:201-206.

Stuart GJ, Sakmann B (1994) Active propagation of somatic action potentials into neocortical pyramidal cell dendrites. Nature 367:69-72.

Takechi H, Eilers J, Konnerth A (1998) A new class of synaptic response involving calcium release in dendritic spines. Nature 396:757-760.

Tsien RW, Lipscombe D, Madison DV, Bley KR, Fox AP (1988) Multiple types of neuronal calcium channels and their selective modulation. Trends Neurosci 11:431-438.

Turrigiano GG, Nelson SB (2000) Hebb and homeostasis in neuronal plasticity. Curr Opin Neurobiol 10:358-364.

Turrigiano GG, Leslie KR, Desai NS, Rutherford LC, Nelson SB (1998) Activity-dependent scaling of quantal amplitude in neocortical neurons. Nature 391:892-896.

Volgushev M, Voronin LL, Chistiakova M, Singer W (1994) Induction of LTP and LTD in visual cortex neurones by intracellular tetanization. Neuroreport 5:2069-2072.

Westenbroek RE, Ahlijanian MK, Catterall WA (1990) Clustering of L-type $\mathrm{Ca}^{2+}$ channels at the base of major dendrites in hippocampal pyramidal neurons. Nature 347:281-284.

Williams SR, Mitchell SJ (2008) Direct measurement of somatic voltage clamp errors in central neurons. Nat Neurosci 11:790-798.

Wyllie DJA, Nicoll RA (1994) A role for protein kinases and phosphatases in the $\mathrm{Ca}^{2+}$-induced enhancement of hippocampal AMPA receptormediated synaptic responses. Neuron 13:635-643.

Wyllie DJA, Manabe T, Nicoll RA (1994) A rise in postsynaptic $\mathrm{Ca}^{2+}$ potentiates miniature excitatory postsynaptic currents and AMPA responses in hippocampal neurons. Neuron 12:127-138.

Yasuda R, Sabatini BL, Svoboda K (2003) Plasticity of calcium channels in dendritic spines. Nat Neurosci 6:948-955.

Yuste R, Denk W (1995) Dendritic spines as basic functional units of neuronal integration. Nature 375:682-684.

Zhang W, Linden DJ (2003) The other side of the engram: experiencedriven changes in neuronal intrinsic excitability. Nat Rev Neurosci 4:885900 . 